\title{
Fractionation protocol design for treatment planning optimization in SIRT using the OEDIPE software
}

\author{
A. Petitguillaume ${ }^{1}$, M. Bernardini ${ }^{2}$, D. Broggio ${ }^{1}$, C. de Labriolle Vaylet ${ }^{3,4}$, D. Franck ${ }^{1}$ and A. Desbrée ${ }^{1 \star}$ \\ 1 IRSN, Laboratoire d'Evaluation de la Dose Interne, 92262 Fontenay-aux-Roses, France. \\ 2 Hôpital Européen Georges Pompidou, Service de médecine nucléaire, 75015 Paris, France. \\ 3 UPMC, Univ. Paris 06 Bio physics, 75005 Paris, France. \\ ${ }^{4}$ Hôpital Trousseau, Service de médecine nucléaire, 75012 Paris, France.
}

Received 4 April 2014 - Accepted 30 July 2014

\begin{abstract}
To go further in the optimization of treatment planning in selective internal radiation therapy (SIRT), radiobiological aspects can be accounted for with the OEDIPE software and used to design fractionation protocols. Dosimetry was performed using data from ${ }^{99 \mathrm{~m}} \mathrm{Tc}-\mathrm{MAA}$ evaluations of 10 patients treated for hepatic metastases with SIRT. The maximal injectable activity (MIA) was calculated, using a tolerance criterion on $\mathrm{BED}_{\text {mean,healthy liver }}$ equal to $54 \mathrm{~Gy}_{2.5}$, for different fractionation protocols, varying the number of fractions, the repartition of activity and the time delay between fractions. OEDIPE was also used to calculate $\mathrm{BED}_{\text {mean }}$ and the EUD to the tumoral liver (TL) that would be delivered with those MIAs. Compared with a single-injection protocol, the MIA is increased on average by $23 \% \pm$ $3 \%, 36 \% \pm 5 \%$ and $45 \% \pm 7 \%$ for fractionation protocols with 2,3 and 4 equal fractions, respectively, while $\mathrm{BED}_{\text {mean, } \mathrm{TL}}$ is increased by $15 \% \pm 2 \%, 23 \% \pm 4 \%$ and $29 \% \pm 5 \%$. $\mathrm{EUD}_{\mathrm{TL}}$, calculated for one evaluation, is increased by $51 \%, 115 \%$ and $159 \%$ using 2, 3 and 4 equal fractions, respectively. For this evaluation, the optimal activity repartition for twofraction protocols is $(3 / 4-1 / 4)$ for time delays of less than 4 days, $(2 / 3-1 / 3)$ for time delays between 4 and 6 days and $(1 / 2-1 / 2)$ for time delays superior to 6 days. Finally, this study confirmed that OEDIPE can be regarded as a tool for treatment planning optimization and fractionation protocol design in SIRT.
\end{abstract}

Keywords: OEDIPE / SIRT / dosimetry / fractionation / treatment planning

\section{Introduction}

Selective Internal Radiation Therapy (SIRT) is an alternative treatment for unresectable hepatic cancers (Dezarn et al., 2011). It consists of the injection of ${ }^{90}$ Y-microspheres, either SIR-Spheres ${ }^{\circledR}$ (SIRTEX) or TheraSpheres ${ }^{\circledR}$ (NORDION), into the hepatic artery, the differences in the vascularization of tumoral and healthy tissues leading to a selective targeting of tumoral lesions. Several steps are required before the microsphere injection. A high-resolution CT scan is first acquired to describe the liver and lesion anatomy precisely. A vascular angiography is then performed to obtain a precise cartography of the liver and lesion vascular beds and to prevent microsphere fixations in unwanted locations using prophylactic embolizations. Technetium human albumin macroaggregates ( ${ }^{99 m}$ Tc-MAA; Pulmocis; Cisbio International), which are supposed to mimic the ${ }^{90} \mathrm{Y}$-microspheres, are then injected and a SPECT/CT scan is acquired to describe the ${ }^{99 \mathrm{~m}} \mathrm{Tc}-\mathrm{MAA}$ spatial distribution.

For SIR-Spheres ${ }^{\circledR}$, the activity to be injected into the patient is then calculated using either body surface area (BSA)

\footnotetext{
^ aurelie.desbree@irsn.fr
}

methods or the partition model (PM) (Ho et al., 1996; Gulec et al., 2006). BSA methods, which are presently used in clinical trials for activity prescription, are based on tumoral and nontumoral liver (TL and NTL, respectively) volumes and on the patient's height and weight without accounting for the quality of the therapy selectivity. On the contrary, the PM is based on the MIRD approach in which limit values on absorbed doses to organs at risk (OARs), i.e. the lungs and NTL for SIRT, are considered to calculate the maximal injectable activity (MIA). In present clinical practice, the PM solely enables one to calculate the activity to be injected into the patient with an approach based on absorbed doses to OARs as recommended by the 2013/59/EURATOM directive from the Council of the European Union (CEU, 2013) for therapeutic applications in nuclear medicine. A recent study (Bernardini et al., 2014) reported large differences between activity prescriptions of BSA methods and the PM. That study highlighted the importance of accounting for the differential of fixation between TL and NTL to both prevent severe toxicities and optimize the injected activity.

However, the absorbed dose calculations used in the PM do not account for the heterogeneity of the activity distribution 
in the regions of interest (ROIs) and for the cross-fire between ROIs. A method, called Personalized Monte Carlo Dosimetry (PMCD), was developed using the OEDIPE software (Petitguillaume et al., 2014b) to overcome these limitations by the use of direct Monte Carlo calculations with patient-specific data on anatomy and activity distribution. OEDIPE was thus used to carry out retrospective dosimetry using data from $14{ }^{99 \mathrm{~m}} \mathrm{Tc}-\mathrm{MAA}$ evaluations of $10 \mathrm{pa}-$ tients treated for hepatic metastases at the Hôpital Européen Georges Pompidou (HEGP, Paris, France) using SIR-Spheres ${ }^{\circledR}$ (Petitguillaume et al., 2014a). The MIA for tolerance criteria based on mean absorbed doses or DVHs were calculated and compared with the PM recommendations. That study demonstrated the potential of personalized Monte Carlo dosimetry for treatment planning optimization in SIRT.

The notion of Biologically Effective Dose (BED) (Dale, 1985), which aims to estimate biological effects induced by an irradiation, was introduced to establish fractionation strategies in external beam radiation therapies (EBRT) and then generalized for brachytherapy and targeted radionuclide therapy. Fractionated protocols enable one to increase treatment efficacy while keeping a constant incidence and severity of toxicity by taking advantage of the differences in radiosensitivity and repair time constants between tumoral and healthy tissues (RCR, 2006). Moreover, the Equivalent Uniform Biologically Effective Dose (EUD) (O'Donoghue, 1999), which enables one to compare heterogeneous absorbed dose distributions with a homogeneous distribution which would have the same biological effects, can be used to assess the levels of potential toxicity and tumor control achieved by a given heterogeneous absorbed dose distribution using, for example, normal tissue complication probability (NTCP) and tumor control probability (TCP) models. Cremonesi et al. (2008) performed BED calculations to retrospectively study the potential of fractionation in SIRT. This study showed that considering multi-cycle treatments could lead to increasing the administered activity with an unvaried BED to NTL, thus demonstrating the potential of fractionation for treatment planning optimization in SIRT. However, those BED calculations were performed using OLINDA/EXM software considering homogeneous distributions of the activity in ROIs. In its latest version, the OEDIPE software enables one to perform BED calculations on the voxel scale from the heterogeneous absorbed dose distribution obtained with the PMCD. These functionalities were thus used to study the potential of fractionated protocols in SIRT when the absorbed dose heterogeneity is taken into account. This paper aims to present the results obtained for fractionated protocols defined varying the number of fractions, the activity distribution among fractions and the time delay between fractions; these protocols being evaluated from the calculation of the BED distribution and EUD that would be achieved with the given protocol and associated MIA.

\section{Materials and methods}

\subsection{The OEDIPE software}

OEDIPE is a user-friendly graphical user interface developed to perform direct Monte Carlo calculations for nuclear medicine applications. Distributions of absorbed dose and BED can be obtained from patient-specific data describing the patient's anatomy and activity distribution. Treatment planning tools are also available to calculate the MIA that meets specified tolerance criteria on absorbed doses or BEDs to OARs for the chosen fractionation protocol. The absorbed dose and BED distribution along with the EUD to ROIs can then be obtained for the MIA and the specified fractionation protocol. OEDIPE's features were extensively described in a previous paper (Petitguillaume et al., 2014b).

\subsection{Application to dosimetry in SIRT}

The latest version of OEDIPE was used to carry out retrospective personalized Monte Carlo dosimetry (3D-PMCD) using data from $14{ }^{99 \mathrm{~m}} \mathrm{Tc}-\mathrm{MAA}$ evaluations of 10 patients treated with SIRT using SIR-Spheres ${ }^{\circledR}$ for hepatic metastases at the Hôpital Européen Georges Pompidou (HEGP, Paris, France). Detailed data on patients' characteristics, on the clinical procedure used to treat these patients at HEGP and on imaging processing were described in previous papers (Bernardini et al., 2014; Petitguillaume et al., 2014a). Regions of interest outlines were drawn on CT images to create patientspecific voxel phantoms using OEDIPE. Normalized 3D matrices of cumulated activity were generated from registered ${ }^{99 \mathrm{~m}}$ Tc-SPECT data. As ${ }^{99 \mathrm{~m}}$ Tc-MAA are injected to predict the ${ }^{90}$ Y-microsphere distribution, predictive absorbed doses on the voxel scale were calculated with the MCNPX Monte Carlo code. For the present study, BED 3D distributions were calculated from these absorbed dose distributions using OEDIPE (Petitguillaume et al., 2014b) with standard values for radiobiological tissue-specific constants (Cremonesi et al., 2008), i.e. $\alpha / \beta=10 \mathrm{~Gy}$ and $\mathrm{T}_{\mathrm{p}}=1.5 \mathrm{~h}$ for hepatic lesions, and $\alpha / \beta=2.5 \mathrm{~Gy}$ and $\mathrm{T}_{\mathrm{p}}=2.5 \mathrm{~h}$ for healthy tissues. These values are not specific to SIRT as, up to now, no values have been derived using clinical data from SIRT treatments. Therefore, other values might be more pertinent in the future to estimate biological effects induced in SIRT treatments.

\subsection{MIA calculations and mean BEDs to ROIs}

Because the notion of BED enables one to compare different therapies, such as external beam radiotherapy (EBRT), targeted radionuclide therapies or brachytherapy, in terms of biological effects, results of nontumoral liver (NTL) irradiation follow-up from external beam radiotherapy were considered to define a tolerance criterion on mean BED to NTL. In this study, this tolerance criterion was thus set to $\mathrm{BED}_{\text {mean,NTL }}=54 \mathrm{~Gy}_{2.5}$, which corresponds to $5 \%$ probability of causing severe hepatitis or liver failure within 5 years (Cremonesi et al., 2008) for standard fractionation protocols delivering a mean absorbed dose to NTL of 30 Gy in 15 fractions of $2 \mathrm{~Gy}$; the " $\mathrm{Gy}_{2.5}$ " unit denotes that the BED has been calculated considering $\alpha / \beta=2.5$ Gy for the NTL. Based on this $\mathrm{BED}_{\text {mean }}$ tolerance criterion, the MIA was calculated for 4 fractionation protocols defined as 1 to 4 fractions with the total activity shared equally among the fractions. These MIAs were calculated from the 3D distribution of BEDs, assuming that the time delay between 

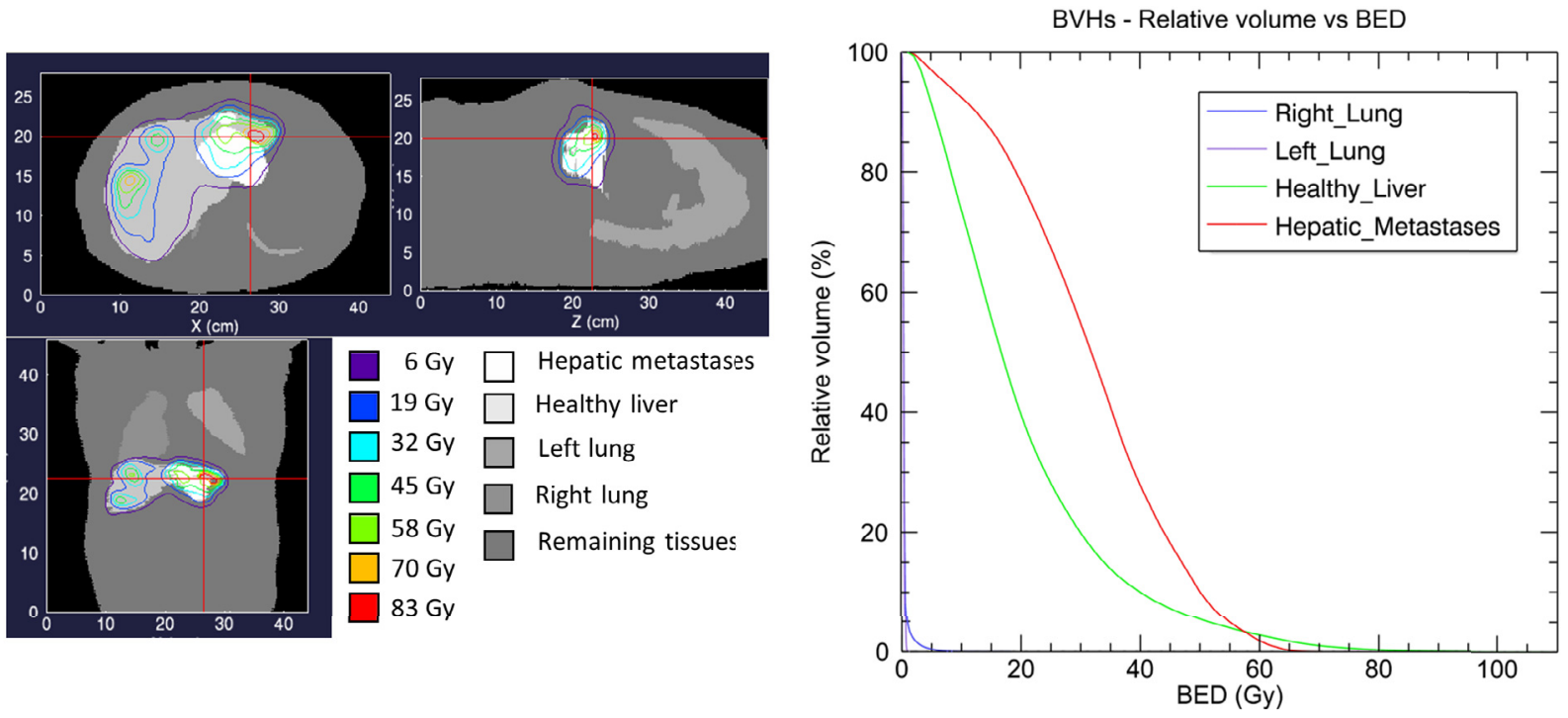

Fig. 1. Results obtained with OEDIPE for evaluation E3 with an injected activity of $1 \mathrm{GBq}$. IsoBED curves (left) superimposed on the voxel phantom and BVHs (right).

fractions is at least ten-fold the radioactive decay half-life, i.e. at least 4 weeks for this application with ${ }^{90} \mathrm{Y}$. OEDIPE was finally used to calculate absorbed doses and BEDs on the voxel scale which could be delivered if these different values of MIA were administered to the patient. Mean absorbed doses and mean BEDs to ROIs, i.e. the lungs, the tumoral liver (TL) and NTL, were obtained along with isodose curves, DVHs, isoBED curves and BVHs.

\subsection{EUD calculations}

For this study, the EUD delivered to TL (EUD ${ }_{\mathrm{TL}}$ ), which is of interest to evaluate tumor control, was calculated using a standard value of $\alpha=0.35 \mathrm{~Gy}^{-1}$ (O'Donoghue, 1999). These calculations were performed for one evaluation (E3) using the BED distribution, obtained considering time delays between fractions of at least ten-fold the radioactive decay half-life, and the MIAs associated with fractionation protocols with 1 to 4 fractions; the total activity shared equally among the fractions.

\subsection{Time delay between fractions}

To study the impact of the time delay between fractions, the BED distribution was calculated for one evaluation (E3) with the equation defining the BED distribution with finite time delays between fractions; this equation was described in a previous paper on the OEDIPE software (Petitguillaume et al., 2014b). The MIA was calculated for two fraction protocols, varying the time delay between fractions from 1 to 28 days and the repartition of the activity among fractions. For these protocols and MIAs, the mean BED to TL $\left(\mathrm{BED}_{\text {mean,TL }}\right)$ was finally calculated using OEDIPE.

\section{Results}

\subsection{Absorbed dose and BED distributions}

IsoBED curves superimposed on the voxel phantom and BVHs for TL and OARs (NTL and both lungs) were obtained for each evaluation. The isoBED curves and BVHs obtained for evaluation E3 with an injected activity of $1 \mathrm{GBq}$ are shown in Figure 1.

\subsection{Fractionation protocols, MIAs and BED mean,TL $_{\text {, }}$}

The values of MIAs obtained for the 4 fractionation protocols with a tolerance criterion on $\mathrm{BED}_{\text {mean,NTL, when } \mathrm{BED}}$ distributions are calculated considering time delays of at least ten-fold the radioactive decay half-life of ${ }^{90} \mathrm{Y}$, are reported in Figure 2a for all 14 evaluations. To quantify the added value of each additional fraction, the percentage of increase in the MIA from one protocol to the next was calculated. On average, using 2 fractions instead of 1 enables one to increase the MIA by $23 \% \pm 3 \%$, using 3 fractions instead of 2 leads to an increase of $11 \% \pm 2 \%$, and using 4 fractions instead of 3 enables one to increase the MIA by $6 \% \pm 1 \%$. Mean BEDs that would be delivered to TL if these MIAs were injected into the patient are reported in Figure $2 \mathrm{~b}$ for all 14 evaluations. On average, using 2 fractions instead of 1 enables one to increase $\mathrm{BED}_{\text {mean,TL }}$ by $15 \% \pm 2 \%$, using 3 fractions instead of 2 leads to an increase of $7 \% \pm 1 \%$, and using 4 fractions instead of 3 enables one to increase $\mathrm{BED}_{\text {mean, } \mathrm{TL}}$ by $5 \% \pm 1 \%$.

\subsection{EUD calculations}

The value of $E D_{\mathrm{TL}}$ obtained for evaluation $\mathrm{E} 3$ for a single-injection protocol was $16.3 \mathrm{~Gy}$. The values of EUD $\mathrm{TL}_{\mathrm{TL}}$ obtained for the same evaluation were 24.7 Gy, 35.0 Gy and 42.2 Gy for fractionated protocols with 2, 3 and 4 fractions, respectively, considering an equal repartition of the MIA among 

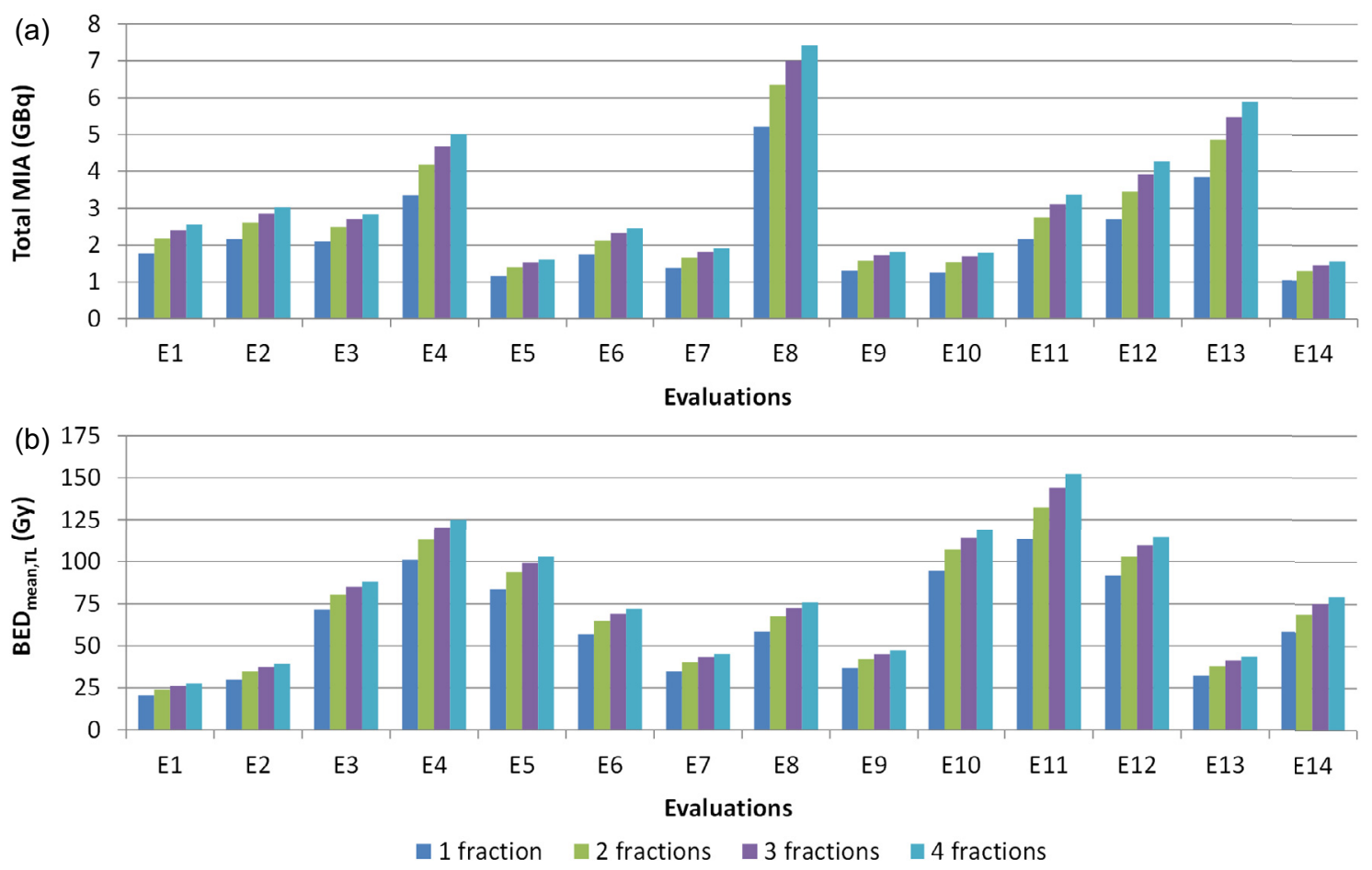

Fig. 2. MIAs obtained for protocols with 1 to 4 fractions (a) and $\mathrm{BED}_{\text {mean,TL }}$ delivered for those MIAs (b).

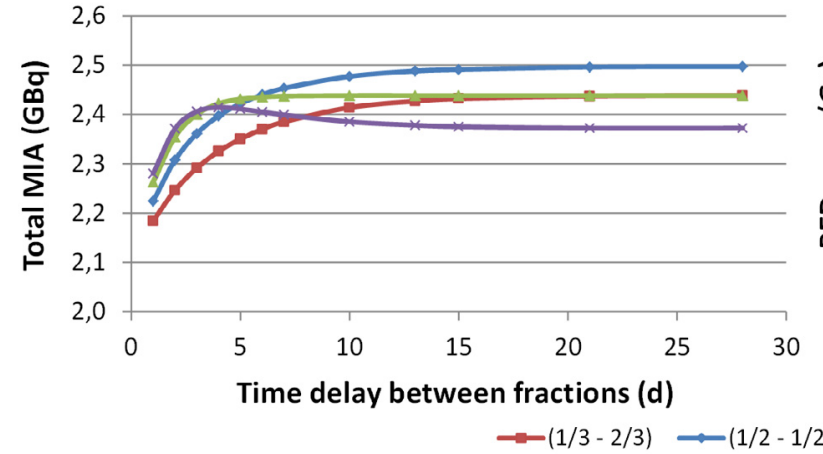

(a)

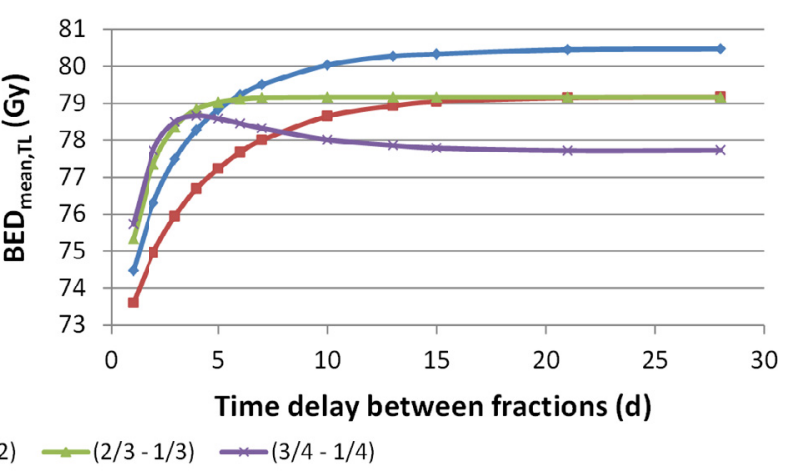

(b)

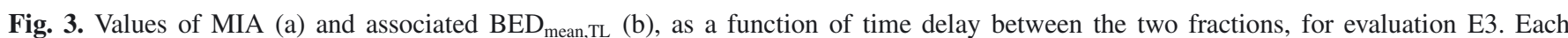
individual curve is related to a particular repartition of the MIA between the two fractions; the (A1 - A2) formalism is related to a protocol with a first injection of activity A1 performed at time equals zero followed by a second injection of activity A2 performed after the time delay represented on the $\mathrm{x}$-axis.

the fractions and time delays between fractions of at least tenfold the radioactive decay half-life.

\subsection{Time delay between fractions}

MIAs for protocols with two fractions were calculated for evaluation E3 considering twelve different time delays between fractions, varying from 1 to 28 days, and four different repartitions of the MIA among the fractions. The MIA and $\mathrm{BED}_{\text {mean, } \mathrm{TL}}$, as a function of the time delay between fractions, are reported in Figures $3 \mathrm{a}$ and $3 \mathrm{~b}$, respectively, each individual curve being related to one repartition of the MIA between the two fractions. Each curve presents a transition phase for time delays of less than 15 days followed by an asymptotic phase reaching the value obtained when the BED distribution is calculated using equation (2). The optimal repartition of the activity between the two fractions depends on the time delay between the two fractions; repartition $(3 / 4-1 / 4)$ is optimal for time delays of less than 4 days, repartition $(2 / 3-1 / 3)$ is optimal for time delays between 4 and 6 days, and repartition $(1 / 2-1 / 2)$ is optimal for time delays of more than 6 days. However, compared with a two-fraction protocol with a time delay of at least ten-fold the radioactive decay half-life, shortening the time delay between the two fractions leads to a de-

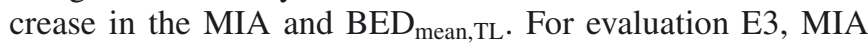
and $\mathrm{BED}_{\text {mean,TL }}$ decreases are inferior to $8.7 \%$ and $5.9 \%$, respectively, compared with the asymptotic values for repartition $(1 / 2-1 / 2)$; these differences fell below $2.5 \%$ for $\mathrm{BED}_{\text {mean,TL }}$ for time delays superior to 3 days. 


\section{Discussion}

The potential of performing a personalized Monte Carlo dosimetry from the ${ }^{99 \mathrm{~m}} \mathrm{Tc}-\mathrm{MAA}$ evaluation for the optimization of treatment planning in SIRT was confirmed in a previous study (Petitguillaume et al., 2014b). With this personalized technique, the differential of fixation between TL and OARs, the heterogeneity of the activity distribution in the ROIs and the cross-fire between the ROIs are accounted for in the calculation of the 3D distribution of absorbed doses. Moreover, because a tridimensional distribution of absorbed doses is available, it can be used in SIRT to calculate the maximal injectable activity (MIA) with different tolerance criteria on OARs to take advantage, for example, of the parallel characteristics of the OARs, i.e. the lungs and NTL. It was also shown that this methodology would be applicable in clinical routine for treatment planning in SIRT, considering computational time, the automation of several steps in OEDIPE and the time delay between the ${ }^{99 \mathrm{~m}} \mathrm{Tc}-\mathrm{MAA}$ evaluation and the ${ }^{90} \mathrm{Y}$-microsphere treatment.

The potential of the radiobiological module implemented in the latest version of OEDIPE was demonstrated in the present study. For given values of radiobiological parameters, it enables one to calculate the BED distribution and the MIA related to tolerance criteria on mean BEDs to OARs for fractionation protocols, along with the EUD and $\mathrm{BED}_{\text {mean,TL }}$ that could be delivered with these protocols. The results obtained for the 14 evaluations and fractionation protocols with 1 to 4 equal fractions show that the higher the number of fractions, the higher the total MIA, the higher the $\mathrm{BED}_{\text {mean,TL }}$, and thus the higher the tumor control, with the same level of toxicity for NTL. For evaluation E3, this point was further highlighted by the fact that the higher the number of fractions, the higher the $\mathrm{EUD}_{\mathrm{TL}}$; compared with a single injection, $\mathrm{EUD}_{\mathrm{TL}}$ is increased by $51 \%, 115 \%$ and $159 \%$ using 2, 3 and 4 fractions, respectively. Moreover, for these fractionation protocols, the results obtained for $\mathrm{BED}_{\text {mean,TL }}$ for the 14 evaluations and for $\mathrm{EUD}_{\mathrm{TL}}$ for evaluation $\mathrm{E} 3$ show that adding a second fraction has more added value than adding a third or a fourth one. Therefore, considering potential risks for the patient in multiple fraction treatments and medical costs, the optimization tool in OEDIPE for fractionation protocols could help medical staff to find the best compromise between tumor control, OAR toxicity and treatment practical feasibility.

Furthermore, for the lesions, the heterogeneity of the absorbed dose distribution might be problematic. In fact, some fractions of the lesions might be under-irradiated while other parts receive a dose superior to the dose threshold for cell killing; the additional dose above this threshold being unhelpful in terms of cell killing. On this point, $\mathrm{EUD}_{\mathrm{TL}}$ is thus of additional added value to evaluate the benefit provided by each additional fraction in terms of tumor control. The small increase in $\mathrm{BED}_{\text {mean, } \mathrm{TL}}$ achieved by the third fraction could lead one to choose a two-fraction protocol. However, in the meantime, the $\mathrm{EUD}_{\mathrm{TL}}$ increase for this third fraction reaches $42 \%$ for evaluation E3 compared with $51 \%$ with the second fraction. Therefore, even if this trend would need to be confirmed by the calculation of $\mathrm{EUD}_{\mathrm{TL}}$ for the other evaluations, this third fraction might be of interest, in terms of tumor control, despite additional risks for the patient and medical costs. Calculations of $\mathrm{EUD}_{\mathrm{TL}}$ for each patient might thus constitute useful additional information to design fractionation protocols.

These results were obtained from BED distributions assuming that the time delay between fractions was at least tenfold the radioactive decay half-life to be able to consider that the residual activity from the previous fractions was negligible. This methodology also assumes that the activity distribution will be identical from one fraction to the next, which might be inadequate with large time delays between fractions due to changes in tissue vascularization, ROI volumes and lesion necrosis over time. New ${ }^{99 \mathrm{~m}}$ Tc-MAA evaluations performed between fractions could be used to update knowledge on the activity distribution. Calculations of absorbed doses and BEDs could then be refined during the course of treatment and used to adjust the MIA for the following fractions.

Considering shorter time delays between fractions could also guarantee a better similarity of the activity distributions between fractions. However, even if the trend would need to be confirmed by running the same study for other evaluations, the results obtained for evaluation E3 for two-fraction protocols with different time delays and activity repartition tend to show that shortening time delays and using unequal frac-

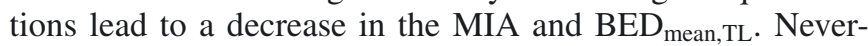
theless, for this evaluation, two-fraction protocols with shorter time delays remain more interesting than a single injection; for evaluation E3, this increase is superior to $8.8 \%$ and $5.7 \%$ (minimum increases obtained for a $24 \mathrm{~h}$ time delay) for the MIA and $\mathrm{BED}_{\text {mean,TL}}$, respectively, compared with the values obtained for a single injection, and reaches $9.5 \%$ and $10.9 \%$ for $\mathrm{BED}_{\text {mean,TL }}$ for time delays superior to 3 and 7 days, respectively. Moreover, the potential of shortening time delays between fractions could be further enhanced by taking into account a repopulation constant for the different ROIs in the calculation of the BED distribution (Dale, 1989). The best number of fractions and time delay between fractions that would keep the absorbed dose rate high enough to compensate for cancerous cell repopulation could then be determined using optimization tools. This potential development could be of particular interest for rapidly growing tumors.

This application of OEDIPE's new functionalities to SIRT demonstrated the theoretical feasibility of treatment planning optimization using absorbed dose and BED calculations for the design of fractionation protocols for targeted radionuclide therapies in general. Due to the technical complexity of SIRT, significantly increasing the number of fractions or shortening time delays between fractions might be difficult for this therapy. In the meantime, it is common practice to use two successive treatments in SIRT (Dezarn et al., 2011). It would thus be of high benefit to perform both accurate calculations of the absorbed doses delivered during the first course of treatment and accurate dosimetry for the second ${ }^{99 \mathrm{~m}} \mathrm{Tc}-\mathrm{MAA}$ evaluation in order to optimize the second course of treatment. Moreover, in terms of treatment efficacy evaluation, the possibility of calculating the EUD is of great interest to consider the establishment of strong dose-effect relationships, which would be independent of the activity distribution heterogeneity, in SIRT or other targeted radionuclide therapies.

For the time being, in the presence of radiopharmaceutical washout, the generation of the $3 \mathrm{D}$ matrix of cumulated activity 
cannot be performed automatically with OEDIPE. However, a new functionality is being developed in OEDIPE either to apply a biokinetic model to the $3 \mathrm{D}$ matrix obtained from registered SPECT data or to model the radiopharmaceutical biokinetics from serial SPECT or PET data. This information on the radiopharmaceutical biokinetics will finally be accounted for in the temporal absorbed dose rate used in the calculation of the BED distribution.

\section{Conclusion}

This study demonstrated that the balance between risks and benefits in SIRT could be further enhanced by taking into account radiobiological aspects through the calculation of the BED distribution. The design of fractionation protocols, depending on a tolerance criterion on the NTL defined from the BED distribution, allows increasing the injected activity and, thus, the absorbed dose and BED to the TL while keeping the level of toxicity to the NTL constant. The calculation of the EUD from the BED distribution can provide additional information to assess the level of toxicity and tumor control. Finally, the establishment of dose-effect relationships from clinical outcomes in targeted radionuclide therapies is strongly needed in order to establish the most pertinent parameter values to be used in the linear-quadratic model and to derive precise tolerance criteria for OARs. The OEDIPE software could be useful in that respect.

\section{References}

Bernardini M., Smadja C., Faraggi M., Orio S., Petitguillaume A., Desbrée A., Ghazzar N. (2014) Selective liver internal radiation therapy with ${ }^{90} \mathrm{Y}$ resin microspheres: comparison between pre-treatment activity calculation methods, Eur. J. Med. Phys. In press.

CEU - Council of the European Union (2013) Council directive 2013/59/EURATOM laying down basic safety standards for protection against the dangers arising from exposure to ionising radiation, and repealing Directives 89/618/Euratom, 90/641/Euratom, 96/29/Euratom, 97/43/Euratom and 2003/122/Euratom, 5 December 2013, p. 25.
Cremonesi M., Ferrari M., Bartolomei M., Orsi F., Bonomo G., Arico D., Mallia A., De Cicco C., Pedroli G., Paganelli G. (2008) Radioembolisation with ${ }^{90} \mathrm{Y}$-microspheres: dosimetric and radiobiological investigation for multi-cycle treatment, Eur. J. Nucl. Med. Mol. Imaging 35, 2088-2096.

Dale R.G. (1985) The application of the linear-quadratic doseeffect equation to fractionated and protracted radiotherapy, $\mathrm{Br}$. J. Radiol. 58, 515-528.

Dale R.G. (1989) Radiobiological assessment of permanent implants using tumour repopulation factors in the linear-quadratic model, Br. J. Radiol. 62, 241-244.

Dezarn W.A., Cessna J.T., DeWerd L.A., Feng W., Gates V.L., Halama J., Kennedy A.S., Nag S., Sarfaraz M., Sehgal V., Selwyn R., Stabin M.G., Thomadsen B.R., Williams L.E., Salem R. (2011) Recommendations of the American Association of Physicists in Medicine on dosimetry, imaging, and quality assurance procedures for ${ }^{90} \mathrm{Y}$ microsphere brachytherapy in the treatment of hepatic malignancies, Med. Phys. 38, 4824-4845.

Gulec S.A., Mesoloras G., Stabin M. (2006) Dosimetric techniques in ${ }^{90} \mathrm{Y}$-microsphere therapy of liver cancer: the MIRD equations for dose calculations, J. Nucl. Med. 47, 1209-1211.

Ho S., Lau W.Y., Leung T.W., Chan M., Ngar Y.K., Johnson P.J., Li A.K. (1996) Partition model for estimating radiation doses from yttrium-90 microspheres in treating hepatic tumours, Eur. J. Nucl. Med. 23, 947-952.

O'Donoghue J.A. (1999) Implications of nonuniform tumor doses for radioimmunotherapy, J. Nucl. Med. 40, 1337-1341.

Petitguillaume A., Bernardini M., Hadid L., de Labriolle-Vaylet C., Franck D., Desbrée A. (2014a) Three-dimensional personalized Monte Carlo dosimetry in ${ }^{90}$ Y-microspheres therapy of hepatic metastases: non tumoral liver and lungs radiation protection considerations and treatment optimization, J. Nucl. Med. 55, 405-413.

Petitguillaume A., Bernardini M., Broggio D., de Labriolle-Vaylet C., Franck D., Desbrée A. (2014b) OEDIPE, a software for personalized Monte Carlo dosimetry and treatment planning optimization in nuclear medicine: absorbed dose and biologically effective dose considerations, Radioprotection 49(4), 275-281.

RCR - The Royal College of Radiologists (2006) Fractionation in radiotherapy: A brief history. In: Radiotherapy dose-fractionation (Board of Faculty of Clinical Oncology, book auth.), pp. 10-13.

Cite this article as: A. Petitguillaume, M. Bernardini, D. Broggio, C. de Labriolle Vaylet, D. Franck, A. Desbrée. Fractionation protocol design for treatment planning optimization in SIRT using the OEDIPE software. Radioprotection 49(4), 283-288 (2014). 